\title{
Listeriosis complicating pregnancy
}

\author{
Vincent Y.T. Cheung MBBS, Wilma L. Sirkin MBBCh
}

Previously published at www.cmaj.ca

A 28-year-old pregnant woman (gravida 2, para 1) presented at 33 weeks' gestation with abdominal cramps and fever. While the cause of her fever was being investigated, continuous fetal heart rate monitoring showed persistent fetal tachycardia with late decelerations. A cesarean section was performed immediately. A male baby was delivered, weighing $2330 \mathrm{~g}$, with Apgar scores of 4,4 and 7 at 1,5 and 10 minutes, respectively. The $\mathrm{pH}$ of the arterial blood in the umbilical cord was 7.04.

The mother was given intravenous ampicillin intraoperatively after the delivery of the baby. The results of a blood culture, which came back on the second postoperative day, showed Listeria monocytogenes. The woman's temperature returned to normal within 48 hours, and she was discharged home on the fourth day after surgery. The woman reported that she had occasionally eaten processed foods without reheating them during pregnancy, but she did not recall eating raw foods or the improper handling of foods.

The infant had decreased tone and reflexes with no respiratory effort at birth, and he requried intubation and ventilatory support. A lumbar puncture was performed, which showed a

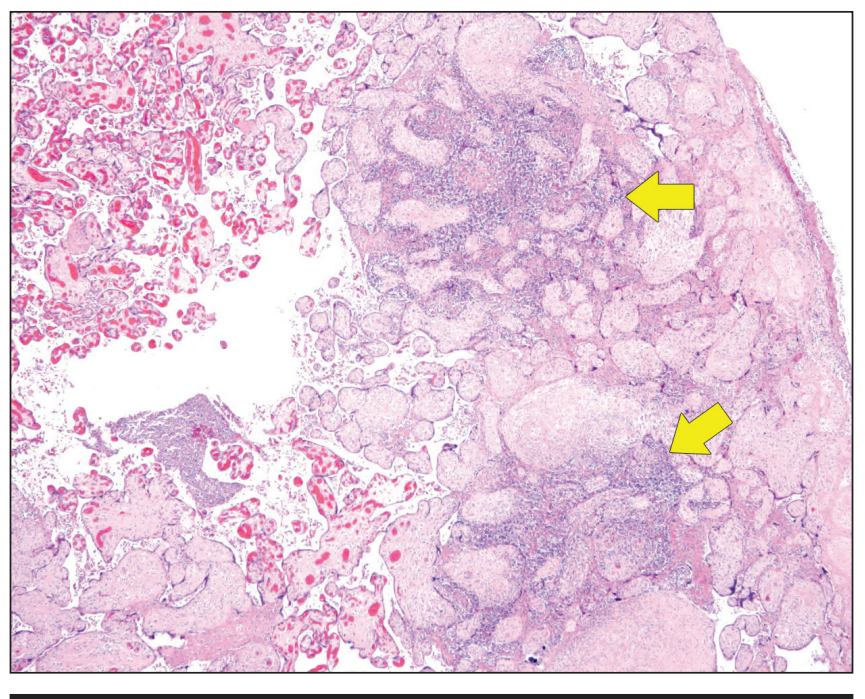

Figure 1: Histology image from the placenta showing acute villitis. The villi are distended and contain numerous acute inflammatory cells. There are multiple microabscesses (arrows) with foci of affected villi showing central necrosis (hematoxylin-eosin stain, original magnification $\times 20$ )

\section{Key points}

- Listeria infection is more common in pregnant women than in nonpregnant women, and may present with fever or flu-like symptoms.

- Although most maternal infections are mild, generalized maternal sepsis can occur and may lead to include miscarriage, preterm delivery and severe fetal and neonatal infection.

- The mortality rate for neonatal infection is up to $50 \%$.

- Most neonates with early-onset listeriosis present with sepsis within 1-2 days of birth.

- In contrast, infants with late-onset listeriosis usually present with meningitis symptoms more than 7 days after birth.

leukocyte count of 22 cells $/ \mathrm{mL}$, with a differential of $24 \%$ polymorphs. the level of protein in the cerebrospinal fluid was 2.2 (normal $0.4-1.2$ ) $\mathrm{g} / \mathrm{L}$, and the level of glucose was 4.1 (2.1-3.6) $\mathrm{mmol} / \mathrm{L}$. The corresponding blood glucose level was $4.2 \mathrm{mmol} / \mathrm{L}$. Culture of the cerebrospinal fluid showed no growth.

The infant was started empirically on broad spectrum antibiotics (intravenous ampicillin and gentamicin). In response to the result of the blood culture taken on day 1 after birth, which showed L. monocytogenes, ampicillin was increased to that used to treat meningitis. The infant had persistent hypotension, which required inotropic support. Between days 2 and 4 after birth, he had several episodes of possible mild seizures that resolved without the need for anticonvulsant therapy. An ultrasound of the infant's head showed mild periventricular edema, which resolved after 4 days. The infant's hemodynamic parameters began to improve. He was gradually weaned off the ventilator and was extubated on day 5 after birth. He was given a 14-day course of antibiotics and was discharged home when he was 30 days old.

Characteristic features of listeriosis were evident on histological examination of the placenta, including severe acute chorioamnionitis, fetal vasculitis and funisitis, and acute villitis with microabscesses (Figure 1). Gram staining showed the presence of gram-positive bacilli (Figure 2).

From the Department of Obstetrics and Gynaecology (Cheung) and the Department of Pathology (Sirkin), North York General Hospital, University of Toronto, Toronto, Ont

Cite as CMAJ 2009. DOI:10.1503/cmaj.090395 


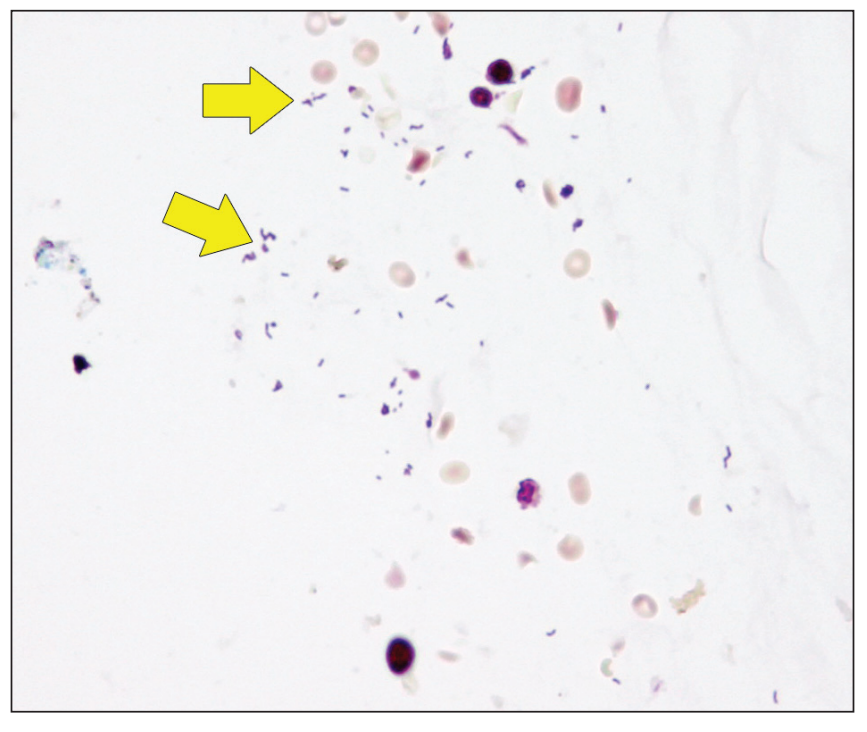

Figure 2: Histology image from the placental membrane showing numerous gram-positive bacilli (arrows) (gram stain, original magnification $\times 600$ ).

\section{Maternal infection}

Infection with L. monocytogenes is rare. However, it occurs more commonly in pregnant women than in nonpregnant women, with an incidence of 12 cases per 100000 pregnant women. ${ }^{1}$ L. monocytogenes is a gram-positive bacillus that has the ability to multiply at refrigerator temperatures $\left(4-10^{\circ} \mathrm{C}\right)$, thus facilitating its transmission through consumption of unpasteurized milk, uncooked meat and vegetables. This infection is particularly dangerous to pregnant women and may result in miscarriage, preterm delivery, generalized sepsis and fetal and neonatal infection. ${ }^{2-4}$ Maternal and fetal outcomes are generally worse if the infection occurs late in gestation. ${ }^{5}$

In most cases of maternal listeriosis, the illness is mild and may be asymptomatic. ${ }^{2-4}$ Fever and flu-like symptoms are the most common presenting symptoms..$^{2-4}$ Often, the definitive diagnosis is made from the results of blood cultures. Ampicillin is the drug of choice for the treatment of listeriosis. For patients allergic to penicillin, erythromycin or vancomycin can be used.

\section{Neonatal infection}

The consequences of neonatal infection are severe, with a mortality rate of $25 \%-50 \% .{ }^{4}$ Early and late forms of neonatal listeriosis have been described. ${ }^{2,3}$ Early-onset listeriosis develops within 1 or 2 days after birth, and most infants show signs of sepsis., ${ }^{2,3}$ A rare severe form of early-onset listeriosis, known as granulomatosis infantisepticum, is characterized by disseminated granulomatous lesions with the formation of microabscesses. ${ }^{1-3}$ Late-onset listeriosis generally occurs more than 7 days after birth, and most infants (94\%) present with meningitis. ${ }^{2,3}$
Ampicillin in combination with an aminoglycoside is the preferred management of neonatal listeriosis. ${ }^{3}$ Cephalosporins have no role because the organism is consistently resistant. If the neonate does not improve within a few days, the addition of rifampicin, moxifloxacin or trimethoprim-sulfamethoxazole may be considered, depending on the sensitivity. ${ }^{2}$

Proper preparation, storage and handling of food is the most effective way to reduce the risk of listeriosis outbreak and, thus, the incidence of fetal and neonatal listeriosis. ${ }^{2-4}$ The Centers for Disease Control and Prevention and the Canadian Food Inspection Agency have made general recommendations to protect against listeriosis. ${ }^{6,7}$ Pregnant women are advised to take additional precautions while consuming and handling foods in order to reduce the chance of maternal infection. ${ }^{6,7}$

This article has been peer reviewed.

Competing interests: None declared.

\section{REFERENCES}

1. Janakiraman V. Listeriosis in pregnancy: diagnosis, treatment, and prevention. Rev Obstet Gynecol 2008;1:179-85.

2. Lorber B. Listeria monocytogenes. In: Mandell GL, Bennett JE, Dolin R, editors Principles and practice of infectious diseases. 6th ed. Elsevier Churchill Livingstone; 2005. p. 2478-84.

3. Bortolussi R, Mailman TL. Listeriosis. In: Remington JS, Klein JO, editors. Infectious diseases of the fetus and newborn infant. 6th ed. W.B. Saunders Company; 2006. p. 465-83.

4. Bortolussi R. Listeriosis: a primer. CMAJ 2008;179:795-7.

5. Cito G, Luisi S, Faldini E, et al. Listeriosis in pregnancy: a case report. J Matern Fetal Neonatal Med 2005;18:367-8.

6. Update: foodborne listeriosis — United States, 1988-1990. MMWR Morb Mortal Wkly Rep 1992;41:251, 257-8.

7. Food safety facts on listeria. Ottawa $(\mathrm{ON})$ : Canadian Food Inspection Agency; 2008. Available: www.inspection.gc.ca/english/fssa/concen/cause/listeriae.shtm (accessed 2009 July 10).

\section{Resources}

\section{General information}

Centers for Disease Control and Prevention

www.cdc.gov/nczved/dfbmd/disease_listing/listeriosis_gi.html

\section{Food safety}

Canadian Food Inspection Agency

www.inspection.gc.ca/english/fssa/concen/cause/listeriae.shtml

\section{Patient brochure for pregnant women}

United States Department of Agriculture Food Safety and Inspection Service

www.fsis.usda.gov/PDF/Protect_Your_Baby.pdf

\section{Clinical guideline}

Ontario Agency for Health Protection and Promotion.

www.health.gov.on.ca/english/providers/pub/disease/listeria /clinical_guidelines_long_20080829.pdf

For more information on listeriosis, see Bortolussi R. Listeriosis: a primer. CMAJ 2008;179:795-7. 